\title{
Shock-induced star formation in a model of the Mice
}

\author{
Joshua E. Barnes \\ Institute for Astronomy, 2680 Woodlawn Drive, Honolulu, HI 96822, USA
}

10 November 2018

\begin{abstract}
Star formation plays an important role in the fate of interacting galaxies. To date, most galactic simulations including star formation have used a density-dependent star formation rule designed to approximate a Schmidt law. Here, I present a new star formation rule which is governed by the local rate of energy dissipation in shocks. The new and old rules are compared using self-consistent simulations of NGC 4676; shockinduced star formation provides a better match to the observations of this system.
\end{abstract}

Key words: galaxies: mergers - galaxies: kinematics and dynamics - galaxies: structure

\section{INTRODUCTION}

Numerical simulations of galaxy formation and interactions often include rules for star formation. In many cases, the local rate of star formation, $\dot{\rho}_{*}$, is related to the local gas density, $\rho_{\mathrm{g}}$, by a power law:

$\dot{\rho}_{*}=C_{*} \rho_{\mathrm{g}}^{n}$,

where $C_{*}$ is a constant. This prescription has been justified both empirically and theoretically. From an empirical perspective, (1) resembles the "Schmidt law" (Schmidt 1959), which relates star formation per unit surface area, $\dot{\Sigma}_{*}$ to gas surface density, $\Sigma_{\mathrm{g}}$; a recent determination of the Schmidt law (Kennicutt 1998) is

$\dot{\Sigma}_{*}=(2.5 \pm 0.7) \times 10^{-4} \frac{\mathrm{M}_{\odot}}{\mathrm{yr} \mathrm{kpc}^{2}}\left(\frac{\Sigma_{\mathrm{g}}}{1 \mathrm{M}_{\odot} \mathrm{pc}^{-2}}\right)^{1.4 \pm 0.15}$

Mihos, Richstone \& Bothun (1991) adopted a rule equivalent to (1) with $n=2$ as an approximation to a Schmidt law with index $\sim 1.8$, while Mihos \& Hernquist (1994a) took $n=1.5$, and presented numerical tests showing this gave a reasonable match to a Schmidt law with index $\sim 1.5$. A more theoretical approach, adopted by Katz (1992) and Springel (2000), sets $\dot{\rho}_{*}=\rho_{\mathrm{g}} / t_{*}$, where $t_{*}$, the time-scale for star formation, is basically proportional to the local collapse time of the gas, $\left(G \rho_{\mathrm{g}}\right)^{-1 / 2}$. This yields (1) with $n=1.5$ and $C_{*}=G^{1 / 2} C_{*}^{\prime}$, where $C_{*}^{\prime}$ is a dimensionless constant.

Despite the apparent convergence of observation and theory on the index $n=1.5$, it's unlikely that (1) really captures the process of star formation. For one thing, only about 1 per cent of the gas actually forms stars per collapse time $\left(G \rho_{\mathrm{g}}\right)^{-1 / 2}$; in other words, consistency with the observations implies that $C_{*}^{\prime} \simeq 10^{-2}$. Current theories of star formation don't offer any straightforward way to calculate

\footnotetext{
* E-mail: barnes@ifa.hawaii.edu
}

this quantity. Gravitational collapse is evidently not the limiting factor which sets the rate of star formation; "feedback" processes are important in determining the fraction of available interstellar material which ultimately becomes stars. While several groups have now devised simulations including star formation regulated by various forms of feedback (e.g. Gerritsen \& Icke 1997; Springel 2000), this approach still has some way to go.

Moreover, models based on (1) don't reproduce the large-scale star formation seen in many interacting galaxies. Mihos. Richstone \& Bothun (1992) found that most of the star formation was confined to the central regions of their model galaxies, while Mihos. Bothun \& Richstone (1993) noted that (1) underestimated the rate of star formation in regions where interstellar gas exhibits large velocity dispersions and gradients, or where the gas appears to be undergoing strong shocks. Mihos \& Hernquist (1994b), Mihos \& Hernquist (1996), and Springel (2000) have modeled the central bursts of star formation seen in ultraluminous infrared galaxies (Sanders \& Mirabel 1996, and references therein). But nuclear starbursts, while a necessary stage in the transformation of merger remnants into elliptical galaxies (e.g. Kormendv \& Sanders 1992), may not be sufficient to accomplish this transformation. For example, merger-induced starbursts create massive young star clusters (e.g. Whitmore \& Schweizen 1995) which may subsequently evolve into globular clusters, but these clusters will be confined to the nuclei of remnants unless the starbursts are spatially extended.

In view of these considerations, it's worth examining alternatives to (1). One long standing idea is that collisions of molecular clouds trigger of star formation (e.g. Scoville, Sanders, \& Clemens 1986). This has been implemented in "sticky particle" schemes which model the interstellar medium as a collection of discrete clouds undergoing inelastic collisions (e.g. Olson \& Kwan 1990; Noguchi 
1991). However, molecular clouds have relatively long mean free paths, and direct collisions between clouds at velocities of $10^{2} \mathrm{~km} / \mathrm{s}$ or more may result in disruption rather than star formation. Jog \& Solomon (1992) proposed a model of shock-induced star formation in interacting galaxies; specifically, they suggested that fast collisions between extended HI clouds create a high-pressure medium which compresses pre-existing molecular clouds and thereby induces bursts of star formation. High-pressure regions, and especially largescale shocks in colliding galaxies, may favor the formation of bound star clusters (Elmegreen \& Efremov 1997).

Recent observations at optical, infrared, and radio wavelengths continue to reveal large-scale star formation in interacting galaxies, including NGC 4038/9, Arp 299, and NGC 4676 (Whitmore \& Schweizer 1995; Vigroux et al. 1996; Mirabel et al. 1998; Hibbard \& Yun 1999; Alonso-Herrero et al. 2000; Xu et al. 2000; de Grijs et al. 2003). Several of these studies explicitly invoke shock-induced star formation in discussing the observational data. In this paper I focus on NGC 4676, a strongly interacting pair of disk galaxies with long tidal tails (Toomre \& Toomre 1972, hereafter TT72; Toomre 1977), as a test-case for models of shock-induced and density-dependent star formation. $\S 2$ describes the star formation algorithms. $\S 3$ presents simulations of NGC 4676, and contrasts the results of shock-induced and densitydependent star formation rules. Conclusions are given in $\S 4$.

\section{SIMULATING STAR FORMATION}

Within the framework of "Smoothed Particle Hydrodynamics" or SPH (Lucv 1977; Gingold \& Monaghan 1977; Monaghan 1992 and references therein), the interstellar material can be modeled as an isothermal gas with a representative temperature $T \sim 10^{4} \mathrm{~K}$. This approach is less complex than others which include radiative cooling (e.g. Barnes \& Hernquist 1996), heating by massive stars (Gerritsen \& Icke 1997), or other feedback effects (Springel 2000). However, previous studies indicate that simple isothermal models capture much of the relevant dynamical behavior of interstellar gas in interacting galaxies, including large-scale shocks, nuclear inflows, and the formation of extended gas disks in merger remnants (Barnes \& Hernquist 1996; Mihos \& Hernquist 1996; Barnes 2002).

With the gas temperature fixed, there remain two interesting local quantities which are easily evaluated in an SPH simulation: the gas density, $\rho_{\mathrm{g}}$, and the rate of mechanical heating due to shocks and $P d V$ work, $\dot{u}$. (An isothermal model assumes that radiative processes locally balance mechanical heating or cooling.) In galactic simulations, the gas temperature is much lower than the virial temperature, so most shocks are highly supersonic and shock heating exceeds $P d V$ work by about two orders of magnitude. Thus shocks can be identified as regions with $\dot{u}$ significantly larger than zero; this criterion serves to locate shock fronts (Barnes 2002 ).

Making use of both $\rho_{\mathrm{g}}$ and $\dot{u}$, I consider a general starformation prescription of the form

$\dot{\rho}_{*}=C_{*} \rho_{\mathrm{g}}^{n} \operatorname{MAX}(\dot{u}, 0)^{m}$, where the MAX function, which returns the larger of its two arguments, is used to handle situations in which $\dot{u}<0$. This prescription is implemented in a probabilistic fashion; the chance of gas particle $i$ undergoing star formation in a time interval $\Delta t$ is

$p_{i}=C_{*} \rho_{i}^{(n-1)} \operatorname{MAX}\left(\dot{u}_{i}, 0\right)^{m} \Delta t$,

where $\rho_{i}$ and $\dot{u}_{i}$, the gas density and heating rate for particle $i$, are defined in Appendix A. At each time-step, $p_{i}$ is computed for each gas particle and compared with a random number $X$ freshly drawn from a uniform distribution between 0 and 1 . If $p_{i}>X$ then the gas particle is instantly and completely converted to a star particle, which subsequently evolves collisionlessly. For later analysis, each star particle formed during a simulation is tagged with the value of the time $t$ at its moment of birth.

While (3) can encompass a wide range of rules, this paper focuses on two limiting cases. First, setting $n>1$ and $m=0$ yields density-dependent star formation, in which $p_{i} \propto \rho_{i}^{(n-1)}$ with no dependence on $\dot{u}$, and regions of higher density produce more stars. Second, setting $n=1$ and $m>0$ yields shock-induced star formation, in which $p_{i} \propto \operatorname{MAX}(\dot{u}, 0)^{m} ;$ star formation is strictly limited to regions with $\dot{u}>0$, which typically correspond to shocks. More general rules with both $n>1$ and $m>0$ are beyond the scope of this paper, but it may be possible to anticipate their behavior using experience gained with the simple cases described here.

Simulations of shock-induced star formation are subject to resolution effects. The width of a shock region in SPH is $\sim 3$ times the local smoothing scale $h_{i}$ (Monaghan 1992) (in these experiments, $h_{i}$ is set to enclose $N_{\text {smooth }}=40$ gas particles within a radius $2 h_{i}$ of each gas particle $i$; see Appendix B). Consider a situation in which a gas particle encounters a shock of some fixed strength. The time that the particle spends in the shock scales as $h_{i}$, while the total amount of energy it dissipates is independent of $h_{i}$, so $\dot{u}_{i} \propto h_{i}^{-1}$. Thus the net probability that the gas particle is transformed into a star particle while passing through the shock is proportional to $h_{i}^{(1-m)}$. The specific choice $m=1$ should produce results which are approximately independent of the spatial resolution. For other choices for $m$ the star formation rate will depend on resolution; to obtain roughly equivalent results in simulations with different spatial resolutions, it will be necessary to adjust the parameter $C_{*}$.

Resolution effects also arise in simulations with densitydependent star formation rules. Such simulations do a good job of reproducing the observed relationship (2) between gas density and star formation rate (Mihos \& Hernquist 1994a; Springel 2000). But current SPH simulations generally don't resolve the vertical structure of gas in galactic disks, so this success may be due, in part, to compensating errors at different distances from the disk midplane. More critically, although shocks with Mach numbers of $M \simeq 10$ to 30 will boost the density of an isothermal gas by factors of $M^{2} \simeq 10^{2}$ to $10^{3}$ (Shu 1992), these high-density regions are not resolved in existing SPH calculations, and the localized bursts of star formation which should result have not been seen in the numerical models. In principle, simulations using density-dependent rules can exhibit shock-induced star formation, but the computing power required to achieve the necessary resolution may not yet be available. 

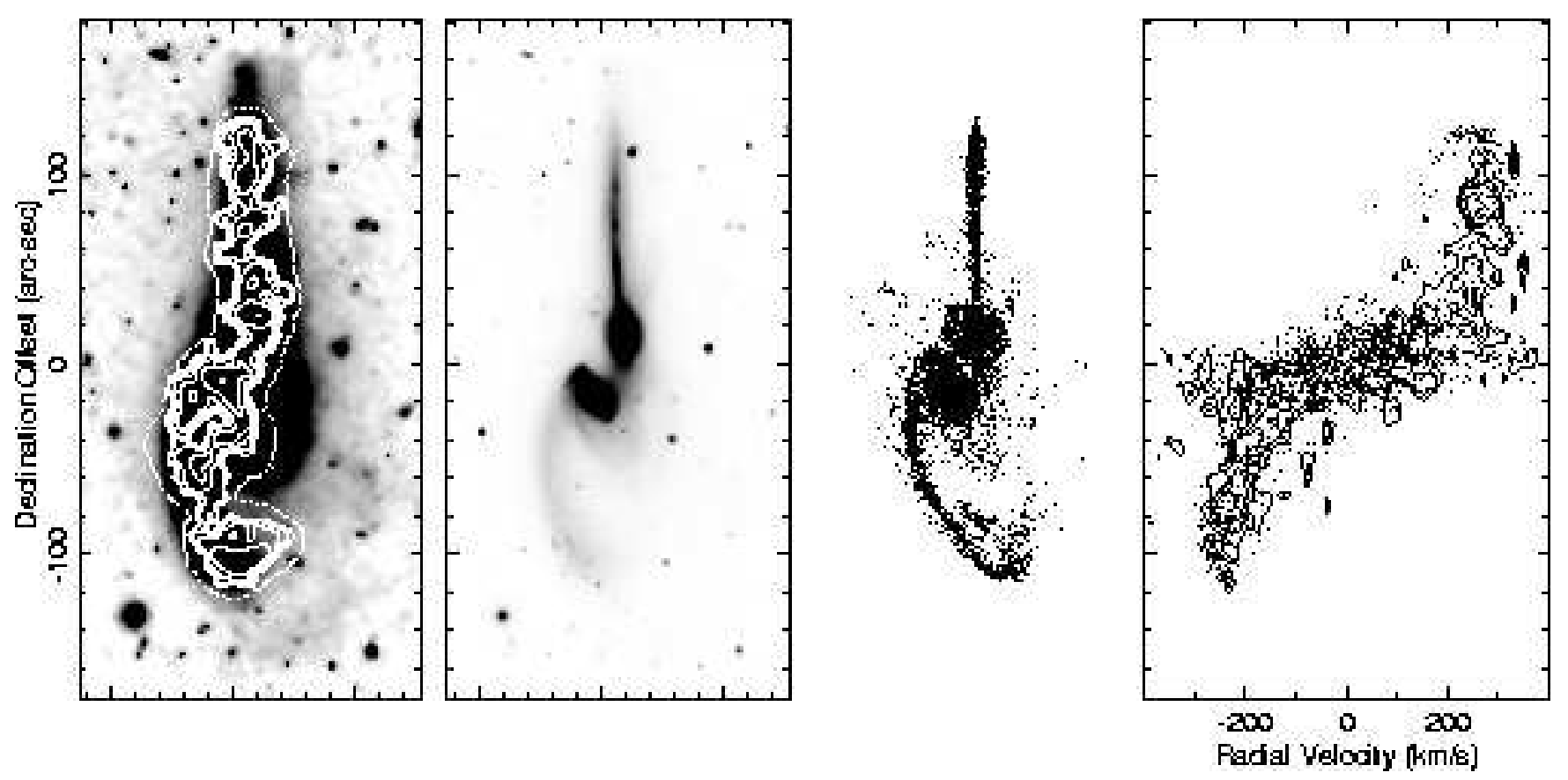

Figure 1. The Mice, NGC 4676, and the model. North is up, west is right. The north-most hulk is NGC 4676a, while its companion to the southeast is NGC 4676b. Far left: deep optical image and HI contours (Hibbard \& van Gorkom 1996). Left: optical image, stretched to show the bodies and tails. Right: self-consistent N-body model. Far right: declination versus line-of-sight velocity plane; contours are HI data, points are N-body model.

\section{MODELING THE MICE}

On account of their rounded bodies and long tails, the two galaxies making up NGC 4676 were dubbed the "Playing Mice" by Vorontsov-Vel'vaminov (1958). The far left panel of Fig. 11 shows a deep optical image, overlain by contours of HI (Hibbard \& van Gorkom 1996), while the adjacent panel shows a shallower image. The individual galaxies are NGC 4676a, to the north, and NGC 4676b, to the southeast. The length and straightness of the bright northern tail imply that we are viewing the aftermath of a nearly-direct passage roughly edge-on to the orbit and disk planes, while the curve of the fainter southern tail is consistent with a more faceon view of an inclined disk. The fairly equal lengths of the two tails suggests that the galaxies involved had roughly equal masses. Along with long-slit data indicating that both "hulks" rotate with north receding (Burbidge \& Burbidge 1961; Thevs. Spiegel. \& Toomre 1972), these considerations led TT72 to a simple model for this system. Their model required that the northern hulk recede faster than the southern one, a prediction soon confirmed with $\mathrm{H} \alpha$ spectroscopy by Stockton (1974). Most kinematic studies, whether based on $\mathrm{H} \alpha$ spectroscopy (Mihos. Bothun \& Richstone 1993), HI interferometry (Hibbard \& van Gorkom 1996), or CO interferometry (Yun \& Hibbard 2001), yield results consistent with Stockton's. On the other hand, H $\alpha$ spectroscopy by Sotnikova \& Reshetnikov (1998) indicated a remarkably large difference of $\sim 300 \mathrm{~km} \mathrm{~s}^{-1}$ between the nucleus of NGC 4676a and its associated tail, and a large velocity dispersion within the tail itself; it's tempting to interpret this as measurement error, but Thevs, Spiegel, \& Toomre (1972) reported somewhat similar results, so the situation is not entirely clear. Nonetheless, HI interferometry appears the best choice for tracing the large-scale dynamics of the system, inasmuch as it provides a full map of the velocity field, and neutral hydrogen is unlikely to be disturbed by star formation.

\subsection{Dynamical model}

The model of NGC 4676 used in this paper is based on work with J. Hibbard (Hibbard \& Barnes, in preparation; see also Barnes 1998). In view of some concerns noted below, this model is somewhat preliminary, but it seems to describe the overall evolution of the system reasonably well.

The initial conditions for the individual galaxies each have three components: a bulge with a shallow cusp (Hernquist 1990), an exponential disk with constant scale height (Freeman 1970; Spitzer 1942), and a dark halo with a constant-density core, based on a "gamma model" (Dehnen 1993; Tremaine et al. 1994). Density profiles for these components are

$\rho_{\text {bulge }} \propto r^{-1}\left(r+a_{\text {bulge }}\right)^{-3}$,

$\rho_{\text {disk }} \propto \exp \left(-R / R_{\text {disk }}\right) \operatorname{sech}^{2}\left(z / z_{\text {disk }}\right)$,

$\rho_{\text {halo }} \propto\left(r+a_{\text {halo }}\right)^{-4}$,

where $r$ is the spherical radius, $R$ is the cylindrical radius in the disk plane, and $z$ is the distance from the disk plane. Numerical results are presented using a system of units with $G=1$. In these units, the components have masses $M_{\text {bulge }}=$ $1 / 16, M_{\text {disk }}=3 / 16, M_{\text {halo }}=1$, and length scales $a_{\text {bulge }}=$ $0.04168, R_{\text {disk }}=1 / 12, z_{\text {disk }}=0.005$, and $a_{\text {halo }}=0.1$. With these parameters, the disk has a rotation period of $\sim 1.0$ time units at $R=3 R_{\text {disk. }}$. Scale factors relating simulation units to real physical quantities will be determined as part of the model-matching process. 
Beginning with TT72's model, we ran experiments systematically varying the relative orbit and orientation of the two identical galaxies. The following parameters produced the reasonably good fit to NGC 4676 shown on the right and far right of Fig. 11 First, the initial orbit was parabolic, with a pericentric separation $r_{\mathrm{p}}=0.25=3 R_{\text {disk }}$. Second, the disks of NGC 4676a and NGC 4676b were initially inclined to the orbital plane by $i_{\mathrm{a}}=25^{\circ}$ and $i_{\mathrm{b}}=40^{\circ}$. Third, the initial angle between each disk's line of nodes and the direction of separation at pericenter was $\omega_{\mathrm{a}}=-30^{\circ}$ and $\omega_{\mathrm{b}}=60^{\circ}$. Fourth, the system was evolved 1 time unit past pericenter. We used an interactive display program to rotate the model to best match the observed morphology and kinematics NGC 4676; further details of the matching process will appear in a subsequent paper.

Comparing the model with the observations, the main discrepancy is the position angle of NGC 4676b's bar; optical images show the bar runs roughly NNE-SSW, while the bar in the model is roughly $\mathrm{N}-\mathrm{S}$ or even NNW-SSE. The pattern speed of a bar depends on the details of the rotation curve, so this discrepancy might be resolved by adjusting the mass model used for NGC 4676b. It's also possible that NGC 4676b's bar predates its encounter with NGC 4676a, in which case the bar's current position angle would depend on its phase at pericenter (Gerin. Combes. \& Athanassoula 1990). However, the hypothesis that NGC 4676b's bar was induced by the tidal interaction is more parsimonious and has not yet been tested by experiments using a range of mass models.

The time yielding the best match between the model and NGC 4676 proved somewhat ambiguous; by adjusting the viewing angle and scaling factors, acceptable matches to the tail morphology and kinematics were obtained for $t \simeq 0.75$ to 1.0 time units after pericenter. At $t=0.875$, a good match was obtained by equating one length unit to $80^{\prime \prime}=35.7 \mathrm{kpc}$, and one velocity unit to $180 \mathrm{~km} \mathrm{~s}^{-1}=0.184 \mathrm{kpc} \mathrm{Myr}^{-1}$. This scaling assumes that NGC 4676's distance is $92 \mathrm{Mpc}$, consistent with $H_{0}=72 \mathrm{~km} \mathrm{~s}^{-1} \mathrm{Mpc}^{-1}$ and NGC 4676's systemic velocity of $6600 \mathrm{~km} \mathrm{~s}^{-1}$ (Hibbard \& van Gorkom 1996). One time unit then works out to $194 \mathrm{Myr}$, and pericenter occurred $\sim 170$ Myr ago. Finally, one mass unit is $2.69 \times 10^{11} \mathrm{M}_{\odot}$.

Given this scaling, the initial parameters of the model galaxies can be computed. For example, the initial disks have reasonable scale lengths $R_{\text {disk }} \simeq 3.0 \mathrm{kpc}$ and masses $M_{\text {disk }} \simeq 5.0 \times 10^{10} \mathrm{M}_{\odot} ;$ on the other hand, they have maximum circular velocities of $\sim 290 \mathrm{~km} \mathrm{~s}^{-1}$, which seem rather high and might be repaired by increasing the halo scale ra$\operatorname{dius} a_{\text {halo }}$.

Since TT72's work, NGC 4676 has been modeled by several other groups. Mihos, Bothun \& Richstone (1993) essentially produced a self-consistent clone of TT72's model, even adopting a short-period elliptical orbit like the one TT72's test-particle methodology dictated; they favored an earlier viewing time which matched the position angle of NGC 4676b's bar but produced tails significantly shorter than those observed. Gilbert \& Sellwood (1993) used Stockton's (1974) velocity data to construct a self-consistent model with a parabolic initial orbit. In most respects, their model is very similar to the one described here, although we favor a closer passage to increase the heft of the tidal tails. Sotnikova \& Reshetnikov (1998) produced a test-particle

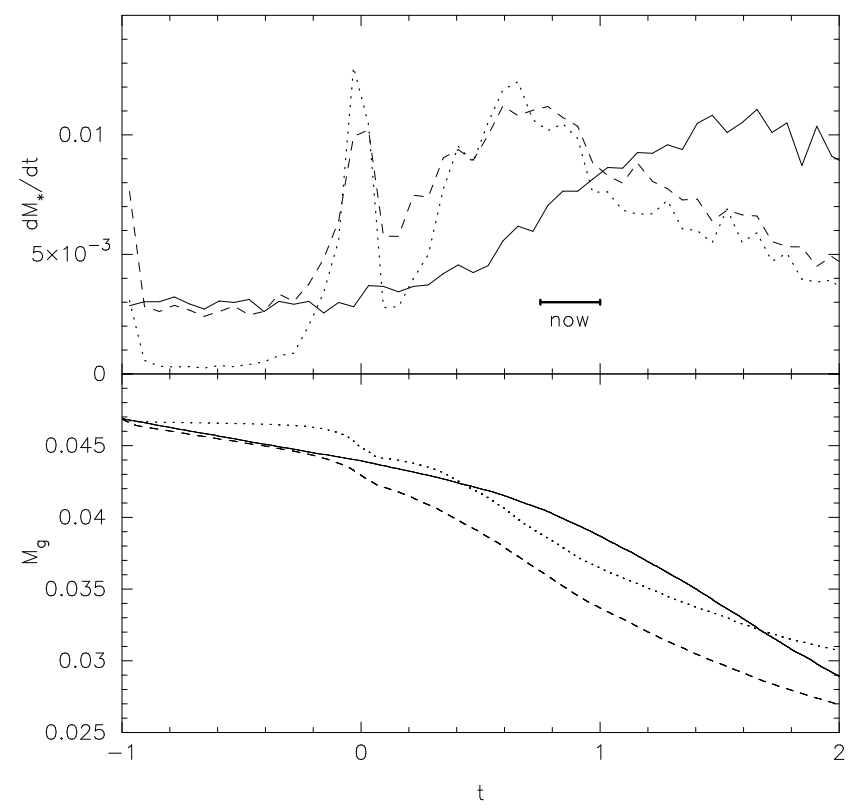

Figure 2. Global star formation in simulations of NGC 4676. The top panel shows $\dot{M}_{*}$, the rate of star formation, while the bottom panel shows the mass of gas remaining; both quantities are given in simulation units $(G=1)$. Pericenter occurred at $t=0$; the horizontal bar labeled "now" indicates the range of times matching the morphology and kinematics of NGC 4676. Solid line: densitydependent star formation with $n=1.5, m=0, C_{*}=0.025$. Broken lines: shock-induced star formation; dashed is $n=1$, $m=0.5, C_{*}=0.5$, dotted is $n=1, m=1, C_{*}=0.25$. Note that the shock-induced models produce strong bursts of star formation at pericenter $(t=0)$, while the density-dependent model exhibits little or no immediate response.

model, restricted to NGC 4676a, which matched the general trend of their velocity data but not the large velocity dispersion they reported within the northern tail. Their model requires relatively massive dark halos to account for these high tail velocities.

\subsection{Interaction-induced star formation}

Fig. 2 compares global star formation rates for three different simulations of NGC 4676. To illustrate the range of possibilities, I contrast one simulation with density-dependent star formation (solid line: $n=1.5, m=0, C_{*}=0.025$ ) and two simulations with shock-induced star formation (dashed line: $n=1, m=0.5, C_{*}=0.5$; dotted line: $n=1, m=1$, $\left.C_{*}=0.25\right)$. All of these simulations were based on the initial orbit and disk orientations described in $\S 3.1$; the gas was initially distributed like the disk stars, and the gas mass in each disk was $M_{\text {gas }}=M_{\text {disk }} / 8=3 / 128$. Further numerical details are given in Appendix B.

In simulations with density-dependent star formation, setting the constant $C_{*}=0.025$ yielded a baseline star formation rate per disk of $\dot{M}_{*} \simeq 0.00136$ (see Appendix C); at this rate, an undisturbed disk would consume its gas on a time-scale of $M_{\text {gas }} / \dot{M}_{*} \simeq 17$ time units $(\sim 3.3 \mathrm{Gyr})$. In the simulation with shock-induced star formation and $m=0.5$, a similar baseline rate of $\dot{M}_{*} \simeq 0.00126$ was obtained by setting $C_{*}=0.5$; this gave reasonable behavior throughout 

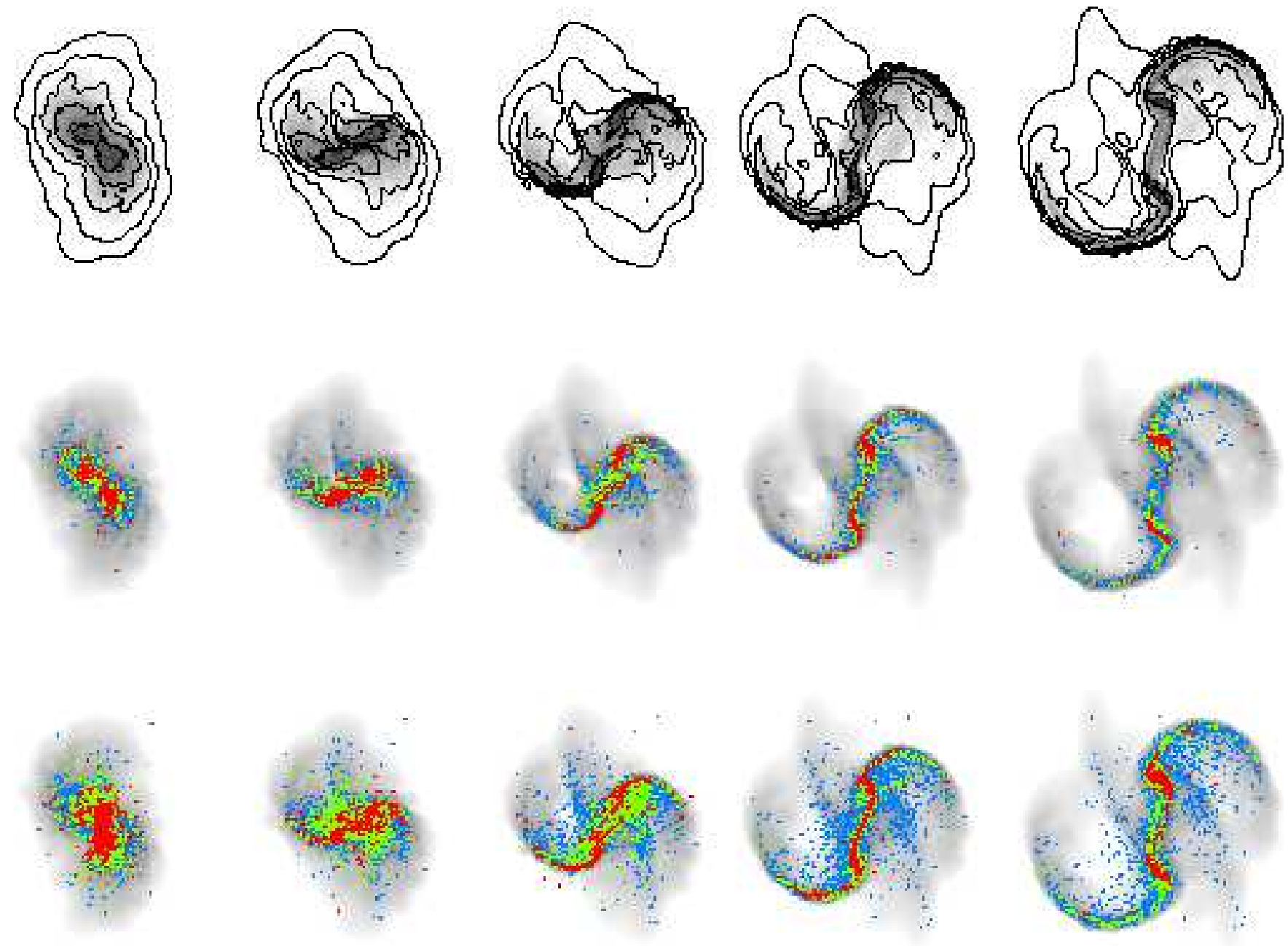

Figure 3. Dynamical evolution and star formation in simulations of NGC 4676. Top row: old stellar distribution (contours) and gas density (halftone) at equally-spaced times between $t=0$ (pericenter) and 0.5. Middle row: old stellar distribution (halftone) and star formation (points) for the density-dependent model $\left(n=1.5, m=0, C_{*}=0.025\right)$. Bottom row: old stellar distribution (halftone) and star formation (points) for the shock-induced model $\left(n=1, m=0.5, C_{*}=0.5\right)$. Red points have ages $\tau<1 / 16$ time units, green have $\tau<1 / 4$, and blue have $\tau<1$. See http://www.ifa.hawaii.edu/ barnes/research/interaction_models/mice/ for animated versions of this and other figures.

the encounter. On the other hand, it was necessary to adopt a much lower baseline rate of $\dot{M}_{*} \simeq 0.000175$ in the simulation of shock-induced star formation with $m=1$ to avoid using up most of the gas in the earliest stages of the encounter; setting $C_{*}=0.25$ insured that this case ultimately consumed about as much gas as the other two.

As Fig. 2] shows, the density-dependent simulation predict that the star formation rate remains fairly constant until some time after the first passage. Eventually, as the gas encountered shocks and gravitationally interacted with the tidally induced bars in the stellar disks, it began accumulating in the centers of the galaxies, driving a fairly gradual increase in star formation. The two shock-induced simulations, on the other hand, produced sharp bursts of star formation as the disks interpenetrated $(t \simeq 0)$, followed by brief lulls as the disks separated. Soon thereafter, star formation rates rose again as gas flows intersected in the perturbed disks; this produced the broad peaks, centered on $t \simeq 0.6$, accounting for most of the stars formed before $t=2$. As one might expect, the simulation with $m=1$ exhibited the most extreme form of this behavior, with a higher peak at $t=0$ and a deeper minimum immediately thereafter.

These various star formation histories have interesting observational implications. According to the densitydependent simulation, star formation in NGC 4676 has just begun responding to the encounter, and the peak star formation rate will occur several hundred Myr from now. In contrast, the shock-induced models predict that we are observing the system a short time after the broad peaks of star formation at $t \simeq 0.6$. This difference in star formation history is fairly insensitive to the choice of $C_{*}$, which influences the overall amplitude of star formation but does little to change the timing of starbursts.

Fig. 3 contrasts the spatial distribution of star formation in density-dependent and shock-induced simulations. The top row of images set the stage by showing distributions of old disk stars and gas, taken from a simulation without star formation. The middle row shows distributions 

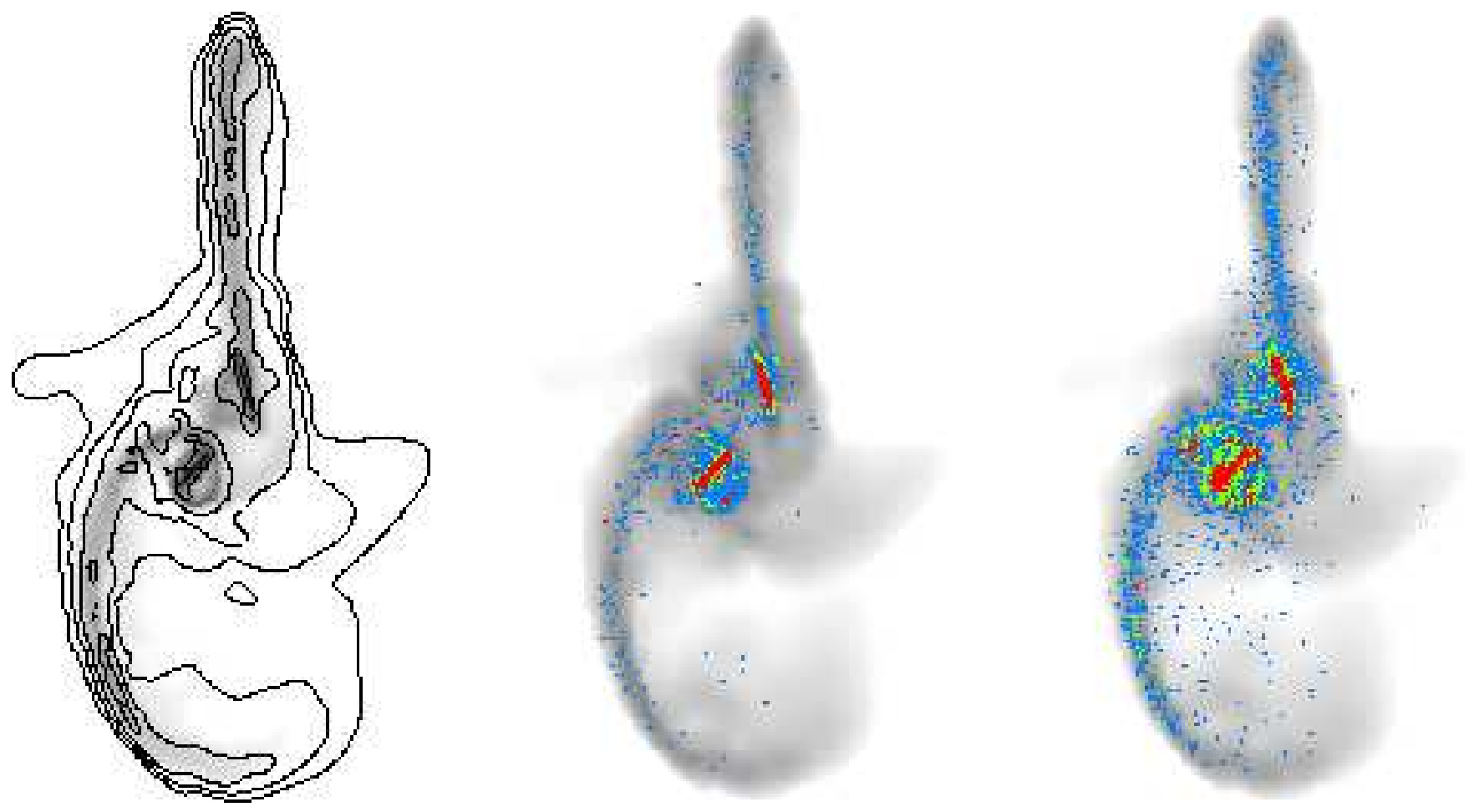

Figure 4. Simulations matching the present configuration of NGC 4676. Left: old stellar distribution (contours) and gas density (halftone). Middle: old stellar distribution (halftone) and star formation (points) for the density-dependent model $\left(n=1.5, m=0, C_{*}=0.025\right)$. Right: old stellar distribution (halftone) and star formation (points) for the shock-induced model $\left(n=1, m=0.5, C_{*}=0.5\right)$. Colors indicate ages as in Fig. [3]

of old disk stars and star particles formed according to the density-dependent rule $\left(n=1.5, m=0, C_{*}=0.025\right)$; colors indicate ages of star particles, with red being youngest and blue oldest. The bottom row presents results for a simulation with shock-induced star formation $(n=1, m=0.5$, $\left.C_{*}=0.5\right)$ using the same color coding. Results for the other shock-induced simulation are not shown since they are very similar.

Comparison of the middle and bottom rows of Fig. 3 shows that the star formation rule clearly influences the distribution of newly-formed stars. In the density-dependent model, star formation was always strongly concentrated toward the central regions of the disks. Some star formation did occur outside the centers, but it was relatively weak and short-lived. In contrast, the shock-induced models produced large, spatially-extended bursts of star formation as the disks collided at $t=0$, and relatively high levels of activity persisted in the bridge and tail regions until $t \simeq 0.4$.

By the last time $(t=0.5)$ shown in Fig. 3 products of star formation are much more widely distributed in the shock-induced simulations than in the density-dependent simulation. Rather interesting in this regard are the gas-poor features which extend to roughly five o'clock from the upper galaxy and to eleven o'clock from the lower galaxy. These regions were swept free of gas during the interpenetrating encounter of the two disks. In the simulations with shockinduced star formation, some of this gas was converted to stars before losing much momentum; as a result, star formed in the pericentric bursts are scattered throughout these gaspoor regions. By contrast, these regions are bereft of both gas and star-formation products in the density-dependent simulation.

Fig. 4 presents views which approximate the observed morphology and kinematics of NGC 4676. These views are plotted at time $t=0.875$, but fairly similar results are found between $t=0.75$ and $t=1$. The simulations shown here are the same ones shown in the last figure, with the density-dependent case in the middle and the shock-induced one on the right. In both cases, most of the ongoing star formation is now confined to central regions of the galaxies. However, products of star formation are found throughout the tails and other tidal features, especially in the simulation with shock-induced star formation. These results invite comparison with observations of past star formation in NGC 4676. Stockton (1974) found that the spectrum of NGC 4676 is dominated by A stars, and attributed this to "rapid, widespread star formation that effectively ceased at least $5 \times 10^{7}$ years ago"; this diagnosis is supported by more recent observations (Mihos. Bothun \& Richstone 1993; Hibbard \& van Gorkom 1996; Sotnikova \& Reshetnikov 1998; de Griis et al. 2003). The shock-induced simulations produced just such bursts at first passage $(t \simeq 0)$, and the products of these bursts are widely distributed throughout the tidal tails at later times. In contrast, the density-dependent simulation had far less star formation outside the galactic centers, and the first passage did not induce a prompt burst of extended star formation.

NGC 4676 has regions of ongoing star formation which are evident in $\mathrm{H} \alpha$ images (Hibbard 1995, Fig. AIII-1). The 
Table 1. Mass of stars formed since start of interaction, expressed as a percentage of initial gas mass.

$\begin{array}{rrrrr}n & m & \text { extended } & \text { central } & \text { total } \\ 1.5 & 0.0 & 1.4 & 9.2 & 10.6 \\ 1.0 & 0.5 & 5.9 & 14.7 & 20.6 \\ 1.0 & 1.0 & 5.2 & 13.6 & 18.8\end{array}$

central regions of the two galaxies are quite prominent in $\mathrm{H} \alpha$ (Mihos. Bothun \& Richstone 1993); central activity is seen in all the simulations. Outside of the centers of the galaxies, star-forming regions are observed in the straight tail extending to the north of NGC 4676a. None of the simulations presented here reproduce this phenomenon, although the shock-induced models come closer inasmuch as they show enhanced star formation in their tails until $t \simeq 0.4$; the density-dependent model seems less promising in this regard since star formation in its tails declines after first passage. $\mathrm{H} \alpha$ images also show ongoing star formation at the base of the curved tail to northeast of NGC 4676b. This region has a relatively high surface brightness and is conspicuously blue in the ACS images (e.g. de Griis et al. 2003). It is nicely reproduced in the shock-induced simulations; gas from the curved tail falls back into its original disk, and the resulting shock sustains a small, coherent patch of extra-nuclear star formation.

On the whole, the simulations with shock-induced rules qualitatively match most of the recent large-scale star formation in NGC 4676. Eventually, this comparison can be made more precise by modeling the spectrophotometric evolution of star particles, but such refinements are beyond the scope of this paper. The balance between central and extended star formation can be quantified by measuring the mass in stars formed since the start of the interaction. Specifically, the production of stars was integrated from $t=-0.25$, which is when Fig. 2 shows the first increase in interaction-induced star formation, until $t=0.875$; particles then found at radii $r>0.15$ from the centers of both galaxies were considered to be part of the extended tidal features. Table 1 list the results for all three experiments. At the epoch matching the present configuration of NGC 4676, the shock-induced models have formed about twice as many stars as the density-dependent model; of course, this ratio depends on the values chosen for $C_{*}$. In the shock-induced simulations, 28 percent of the newly-formed stars are found within the tidal features, while the corresponding figure for the density-dependent simulation is only 13 percent. These percentages should be insensitive to reasonable variations of $C_{*}$, and preliminary experiments with other parameter values support this expectation.

\subsection{Starbursts in mergers}

The future evolution of the NGC 4676 system may seem a matter of conjecture. Nonetheless, it's worth asking when these galaxies are likely to merge and if their merger will produce an ultraluminous IR galaxy; even if we can't check the specific predictions for this system, the results may provide insight into other merging galaxies. I therefore present some predictions for the fate of NGC 4676.

Fig. 15 extends the comparison of global star formation rates in NGC 4676 some $600 \mathrm{Myr}$ into the future. In these simulations, star formation is modulated by two key events.

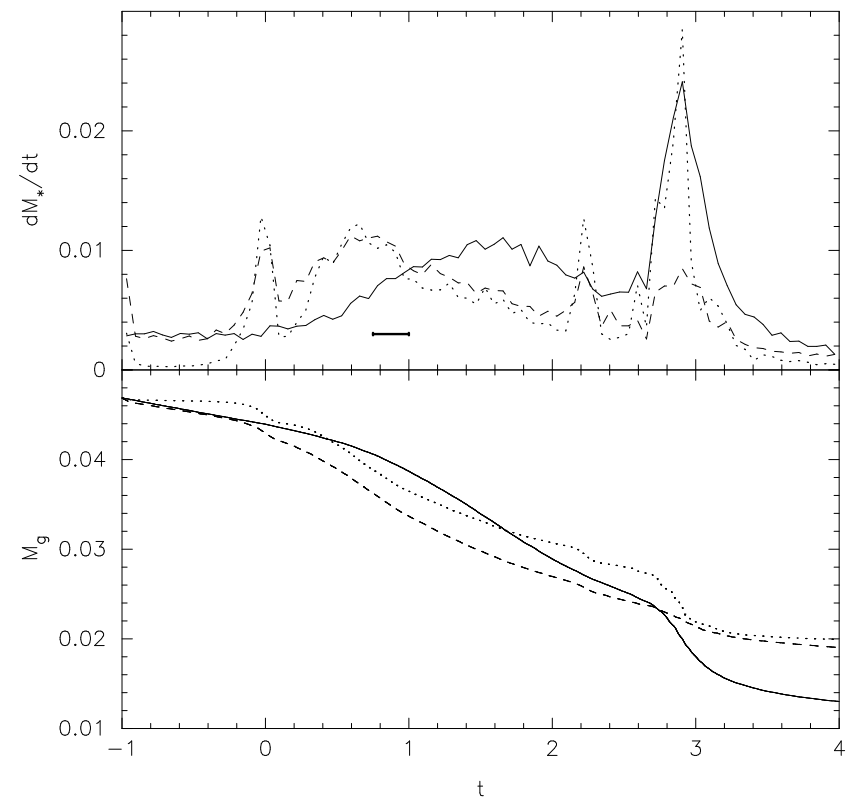

Figure 5. Global star formation in simulations of NGC 4676 . First pericenter occurs at $t=0$, second pericenter occurs at $t \simeq$ 2.2 , and the galaxies merge at $t \simeq 2.8$. Line types are identical to those in Fig. 2

First, as a result of orbital decay, the galaxies have a second and much closer passage at $t \simeq 2.2$; this passage has little immediate effect in the density-dependent model, but produces definite bursts in the shock-induced models, especially in the one with the steeper $m=1$ dependence on $\dot{u}$. Second, the galaxies merge at $t \simeq 2.8$, producing dramatic bursts of star formation in the density-dependent model and in the shock-induced model with $m=1$, but only a modest burst in the shock-induced model with $m=0.5$.

In the shock-induced simulations, the bursts at the second passage were produced by the same mechanism responsible for the comparable, albeit somewhat larger, bursts at first pericenter: interpenetrating encounters between two gassy disks. While much of the gas originally populating these disks was driven into the nuclei or converted into stars, gas returning from the tidal tails settled back into the disks (e.g. Barnes 2002); thus, even after the fireworks attending their first passage, the disks had enough extended gas to produce brief starbursts as they came together for a second time. It's worth noting that such "second-passage" activity is apparently occurring in Arp 299, which exhibits both an extended starburst only 4 Myr old (e.g. Alonso-Herrero et al. 2000) and a long tidal tail with an age of $\sim 750$ Myr (Hibbard \& Yun 1999).

After the second passage, the orbits of the galaxies decayed very rapidly, and a final, almost head-on encounter resulted in merger at about $t=2.8$. As noted above, this event produced fairly intense bursts of star formation in two of the three cases studied here. In the density-dependent simulation, this burst resulted from a sudden increase in gas density as the nuclei finally coalesced. Similar results have been seen in other numerical studies using densitydependent star formation; Mihos \& Hernquist (1996) report bursts with peak star formation rates as much as $10^{2}$ times 
Table 2. Log median radii of burst populations.

$\begin{array}{rrrrrrr}n & m & \mathrm{~B}_{1} & \mathrm{~B}_{2} & \mathrm{~B}_{3} & \mathrm{~B}_{4} & \text { all } \\ 1.5 & 0.0 & & -1.57 & & -2.44 & -1.78 \\ 1.0 & 0.5 & -0.55 & -1.10 & -1.34 & -1.91 & -1.23 \\ 1.0 & 1.0 & -0.49 & -1.10 & -1.09 & -1.85 & -1.30\end{array}$

Table 3. Mass of burst populations, expressed as a percentage of initial gas mass.

$\begin{array}{rrrrrrr}n & m & \mathrm{~B}_{1} & \mathrm{~B}_{2} & \mathrm{~B}_{3} & \mathrm{~B}_{4} & \text { all } \\ 1.5 & 0.0 & & 29.9 & & 16.6 & 72.1 \\ 1.0 & 0.5 & 5.1 & 14.1 & 3.2 & 4.9 & 59.5 \\ 1.0 & 1.0 & 4.7 & 13.9 & 4.1 & 11.3 & 57.6\end{array}$

the baseline. The present calculation yielded a more modest burst, peaking a factor of $\sim 8$ above the baseline; the lower peak in the present simulation may be due to consumption of gas at times $t \simeq 1$ to 2 while the galaxies were still separate. Springel (2000), combining density-dependent star formation with a sophisticated feedback scheme, obtained final bursts comparable to the one shown here. On the other hand, Mihos, Bothun \& Richstone (1993) reported only a "weak merger-induced" starburst in their model of NGC 4676; while they blame this fizzle on the long delay between first passage and merger in their dynamical model, it's possible that the steeper $n=2$ exponent of their star formation prescription had some role in consuming almost all available gas before the final encounter.

Up until this point, the two simulations with shockinduced star formation have produced fairly similar results. Nonetheless, as Fig. 5 shows, their final bursts are quite different; the $m=0.5$ model yields a small burst with a maximum $\dot{M}_{*} \simeq 0.0085$, while the $m=1$ model yields a sharply-peaked burst with a maximum $\dot{M}_{*} \simeq 0.0285$. In principle, this difference could originate at earlier times for example, the $m=0.5$ model might yield a smaller final burst because it had already used up its gas - but a detailed examination of the simulations does not support this idea. At the start of their final encounters, both simulations have comparable amounts of high-density nuclear gas, and in both simulations these central regions collided almost headon, producing comparable amounts of dissipation. Thus, the difference in burst strengths reflects the different behavior of the $m=0.5$ and $m=1$ rules under nearly identical circumstances: in both cases, a moderate amount of gas encountered a strong shock as the nuclei collided. The steeper dependence on $\dot{u}$ in the $m=1$ case converted a large fraction of the shocked gas into stars, while in the $m=0.5$ case the probability of star formation is less strongly enhanced and less gas was consumed. While the final bursts are not as dramatic as those reported by Mihos \& Hernauist (1996), it's possible that further simulations of shock-induced star formation with $m=1$ could produce bursts large enough to power luminous IR galaxies.

Tables 2 and 3 summarize properties of the starbursts produced in these simulations. Here a burst is defined as all stars formed in a certain time interval; these intervals bracket various peaks of $\dot{M}_{*}$ in Fig. [5] For the shock-induced simulations, burst " $\mathrm{B}_{1}$ " corresponds to $-0.20 \leqslant t \leqslant 0.12$, " $\mathrm{B}_{2}$ " to $0.28 \leqslant t \leqslant 0.93$, "B $\mathrm{B}_{3}$ " to $2.10 \leqslant t \leqslant 2.34$, and " $\mathrm{B}_{4}$ " to $2.69 \leqslant t \leqslant 3.00$. For the density-dependent simulation, " $\mathrm{B}_{2}$ " corresponds to $0.80 \leqslant t \leqslant 2.34$, and " $\mathrm{B}_{4}$ " to $2.69 \leqslant$ $t \leqslant 3.15$. In effect, bursts $\mathrm{B}_{1}$ and $\mathrm{B}_{3}$ occur when the galaxies interpenetrate at first and second passages, respectively, $\mathrm{B}_{2}$ is the broad peak of star formation in the individual galaxies after their first passages, and $\mathrm{B}_{4}$ is the final merger-induced starburst. The last column in each table, labeled "all", gives figures for all stars formed during these simulations $(-1 \leqslant$ $t \leqslant 4)$.

The radial distribution of burst populations in the final $(t=4)$ merger remnants is characterized in Table 2 Here, the center of each remnant $\mathbf{r}_{\text {cent }}$ was defined by locating the minimum of the potential well; next, the median of $\left|\mathbf{r}_{i}-\mathbf{r}_{\text {cent }}\right|$ for all particles $i$ in each burst population was computed; common logarithms of these median radii are listed. On the whole, the burst populations in the shockinduced simulations are $\sim 3$ times more extended than those in the density-dependent simulation; this ratio applies not only to the stars produced by bursts $\mathrm{B}_{2}$ and $\mathrm{B}_{4}$ but also to the entire population of stars formed during the simulations. The extended distribution of star formation products in the shock-induced models may provide a better match to galaxies like NGC 7252, where Schweizer (1990) found spectra dominated by A stars both within the nucleus and at a projected distance of $7 \mathrm{kpc}$. In addition, the final $\mathrm{B}_{4}$ populations in the shock-induced simulations, while still compact, may be extended enough to moderate the central "spikes" of young stars that Mihos \& Hernquist (1994c) found in their simulated merger remnants.

Table 3 lists masses of stars formed. These masses are given as percentages of the total initial gas mass, $2 M_{\text {gas }}=$ 0.046875 . The two shock-induced simulations, by design, formed comparable amounts of stars, while the densitydependent simulation ultimately formed somewhat more. Burst $\mathrm{B}_{2}$, triggered within the individual galaxies after their first passage, yielded 41 percent of all stars formed in the density-dependent model, but only 24 percent in the shockinduced models. The final burst $\mathrm{B}_{4}$ accounts for between 8.2 percent and 23 percent of the total star formation.

Earlier merger simulations including shock-induced star formation have largely been based on "discrete cloud" models of the ISM, making a direct comparison with the present results difficult. Noguchi (1991) presented cloud-collision rates for several mergers; assuming that each cloud collision resulted in star formation, he predicted that merging galaxies undergo "repetitive starbursts" as the relative orbit of their nuclei decays. The present calculations also produce bursts of star formation associated with pericentric passages, but orbit decay in these fully self-consistent calculations is much more abrupt than in Noguchi's experiments, possibly because the self-gravity of the gas is included. Thus, while there is a good deal of temporal structure in the star formation rate, the nearly-periodic starbursts Noguchi reported, which seem to reflect the gradual decay of elongated orbits in a nearly-harmonic core, are not seen here. Olson \& Kwan (1990) modeled the evolution of a spectrum of cloud masses during a merger, and reported a dramatic increase in the rate of disruptive cloud-cloud collisions within the inner $2 \mathrm{kpc}$ of their model galaxies. Assuming that star formation is associated with disruptive and glancing cloud-cloud collisions, they obtained peak luminosities and luminosity-to-gas ratios typical of luminous IR galaxies. 


\section{CONCLUSIONS}

Density-dependent and shock-induced models of star formation yield qualitatively and quantitatively different results. These differences arise despite the fact that the gas density $\rho_{\mathrm{g}}$ and the dissipation rate $\dot{u}$ are not completely independent variables; indeed, they can be explained in terms of the global relationship between $\rho_{\mathrm{g}}$ and $\dot{u}$. Dissipation is a necessary precursor for any significant increase in central gas density, since gas can only accumulate in galactic centers as a result of an irreversible process. The rate of increase in gas density closely tracks the net energy radiated away in shocks (Barnes \& Hernquist 1996, Fig. 6); globally, $\dot{u}$ and $d \rho_{\mathrm{g}} / d t$ are strongly correlated. Thus, models of shock-induced star formation can respond promptly to external disturbances, while little activity occurs in density-dependent models until $\rho_{\mathrm{g}}$ has had time to build up. The larger spatial extent of star formation in shock-induced models is, in part, a corollary of the earlier onset of activity in such models, since the gas is more widely distributed at earlier times.

Density-dependent rules may be used to implement unified models of star formation in normal and interacting galaxies. The disks of normal galaxies and the central regions of starburst galaxies fit onto the same power law (Kennicutt 1998). Thus, by setting $C_{*}$ to match the baseline rate of star formation in unperturbed disks, Mihos \& Hernquist (1996) were able to produce starbursts comparable to those inferred in ultraluminous infrared galaxies (Sanders \& Mirabel 1996); this represents a real success for the model defined by (1) with $n=1.5$.

But there is abundant evidence for large-scale star formation in interacting galaxies. This includes ongoing star formation in the "overlap regions" of systems like NGC 4038/9 and Arp 299, as well as the $\mathrm{H} \alpha$ emission from the tails of NGC 4676. It also includes the A-star spectra seen in the tails of NGC 4676 and throughout the body of NGC 7252. Density-dependent star formation can't easily explain these observations; interactions funnel gas from the disks to the central regions of galaxies, thereby promoting rapid star formation in galactic nuclei but reducing the supply of gas needed for star formation elsewhere. Moreover, violent relaxation ceases long before binding energies are effectively randomized (e.g. White 1979; Barnes 1992), so merging is ineffective at transporting the products of star formation outward from nuclei to the bodies of merger remnants. Thus, density-dependent rules offer at best an incomplete description of star formation in interacting galaxies.

In simulations with shock-induced rules, the star formation rate depends on dynamical circumstances. Shocks in unperturbed disks are associated with transient spiral patterns, and matching the baseline level of star formation depends on reproducing the "right" level of spiral structure. This, in itself, is a tough problem, and it's not clear that shock-induced models will soon yield a unified description of star formation in normal and interacting galaxies. Until this becomes possible, unperturbed disks can't be used to set $C_{*}$, so the amplitude of a shock-induced starburst can't be predicted a priori. Nonetheless, shock-induced star formation is an important element of galactic collisions, and its implementation in numerical simulations is a useful step toward increased realism.

These simulations have contrasted two limits of (3): density-dependent star formation, with $n>1$ and $m=0$, and shock-induced star formation, with $n=1$ and $m>0$. These cases were chosen as instructive examples, but (3) is general enough to accommodate additional possibilities. First, setting $n>1$ and $m$ just slightly larger than 0 would yield a modified law in which star formation is proportional to $\rho_{\mathrm{g}}^{n}$ but occurs only in regions with $\dot{u}>0$; this resembles the rule adopted by Katz (1992), who basically took $\dot{\rho}_{*} \propto \rho_{\mathrm{g}}^{3 / 2}$ but restricted star formation to regions with convergent flows. Notice that cases with $0<m \ll 1$ are not continuous with the case $m=0$; in the latter, star formation is strictly independent of $\dot{u}$. Second, setting $n>1$ and $m>0$ would yield hybrid rules in which star formation depends on both $\rho_{\mathrm{g}}$ and $\dot{u}$. The consequences of such rules can sometimes be inferred from the limiting cases considered here. For example, in the simulations of NGC 4676 with shockinduced star formation, the gas involved in burst $\mathrm{B}_{3}$ has densities $\sim 10^{2}$ times higher than the gas involved in burst $\mathrm{B}_{1}$; setting $n>1$ would boost $\mathrm{B}_{3}$ relative to $\mathrm{B}_{1}$ by a factor of $\sim 10^{2(n-1)}$, assuming that neither burst was limited by the supply of gas.

Perhaps most exciting are the new avenues for research created by an alternative description of star formation in galaxy interactions:

- In contrast to density-dependent star formation, which seems fairly insensitive to most details of galactic encounters (Mihos \& Hernquist 1996), different encounter geometries may yield a variety of shock-induced star formation histories. A survey of different encounters, along the lines of other surveys without star formation (e.g. Barnes 2002), might establish if widespread star formation at first passage is generic or limited to a subset of close encounters, and also determine if shock-induced star formation can account for ultraluminous infrared galaxies.

- Shock-induced star formation makes definite predictions about the timing of starbursts triggered by galactic encounters. As already mentioned, models including the photometric evolution of starburst populations could sharpen the comparison between simulations and observations of systems like NGC 4676. In addition, these models might predict the ages of embedded young star clusters; such predictions could be checked by multi-object spectroscopy of the clusters.

- In the context of merger simulations, shock-induced star formation also yields predictions for the spatial distribution and kinematics of starburst populations. It would be quite interesting to compare these predictions with observations of the distribution and kinematics of metal-rich globular clusters in elliptical galaxies, which may have been formed in merger-induced starbursts (e.g. Zepf \& Ashman 1993).

- Encounters between gas-rich galaxies at redshifts of a few seem a natural setting for shock-induced star formation. While many high- $z$ galaxies have peculiar morphologies (e.g. van den Bergh et al. 1996), the bridges and tails characteristic of low- $z$ encounters (TT72) are not very evident. Instead, the optical morphology of these objects may be dominated by rest-frame UV from widespread starbursts; if so, the photometric modeling approach described above could help interpret existing and forthcoming observations of interacting galaxies at intermediate and high redshifts. 


\section{ACKNOWLEDGMENTS}

I thank John Hibbard for permission to discuss our modeling of NGC 4676 in advance of publication, and for helpful discussions. I also thank Chris Mihos, Lars Hernquist, and especially Francois Schweizer for comments on this paper, and the referee for a prompt and helpful review.

\section{APPENDIX A: SPH EQUATIONS}

I use an adaptive SPH code in which each gas particle $i$ is assigned smoothing length $h_{i}$ which depends on local conditions. The gas density at particle $i$ is estimated using the symmetric form suggested by Hernquist \& Katz (1989),

$\rho_{i}=\sum_{j} m_{j} \bar{W}_{i j}\left(r_{i j}\right)$,

where $m_{j}$ is the particle mass and $r_{i j} \equiv\left|\mathbf{r}_{i}-\mathbf{r}_{j}\right|$. Here, $\bar{W}_{i j}(r) \equiv \frac{1}{2}\left[W\left(r, h_{i}\right)+W\left(r, h_{j}\right)\right]$, and $W(r, h)$ is the spline interpolation kernel (Monaghan \& Lattanzio 1985), which vanishes identically for $r>2 h$.

As discussed in $\S 2$, the code uses an isothermal equation of state, so the internal energy $u_{i}$ of each gas particle is constant. One may nonetheless compute the mechanical heating rate $\dot{u}_{i}$ associated with the pressure and (artificial) viscous forces on each particle $i$, and assume that this heating is exactly balanced by radiative processes. This requires only a modest number of computations over and above those required to compute the forces themselves, and allows a global check of energy conservation. The mechanical heating rate is estimated using

$\dot{u}_{i}=\sum_{j \neq i} m_{j}\left(\frac{P_{i}}{\rho_{i}^{2}}+\frac{\Pi_{i j}}{2}\right)\left(\mathbf{v}_{i}-\mathbf{v}_{j}\right) \cdot \frac{\partial}{\partial \mathbf{r}_{i}} \bar{W}_{i j}\left(r_{i j}\right)$,

where $P_{i}=c_{\mathrm{s}}^{2} \rho_{i}$ is the pressure at particle $i$ for an isothermal gas with sound speed $c_{\mathrm{s}}$. Here, $\Pi_{i j}$ approximates a combination of bulk and von Neuman-Richtmyer artificial viscosity (Monaghan 1992),

$\Pi_{i j}=\left\{\begin{array}{cc}\frac{-\alpha c_{\mathrm{s}} \mu_{i j}+\beta \mu_{i j}^{2}}{\left(\rho_{i}+\rho_{j}\right) / 2}, & \mu_{i j}<0 ; \\ 0 & \text { otherwise }\end{array}\right.$

where $\alpha$ and $\beta$ are parameters of order unity,

$\mu_{i j}=\frac{\left(\mathbf{r}_{i}-\mathbf{r}_{j}\right) \cdot\left(\mathbf{v}_{i}-\mathbf{v}_{j}\right)}{r_{i j}^{2} / h_{i j}+\eta^{2} h_{i j}}$,

$h_{i j} \equiv \frac{1}{2}\left(h_{i}+h_{j}\right)$, and $\eta$ is a parameter of order $10^{-2}$.

\section{APPENDIX B: SIMULATION DETAILS}

The disk galaxies used in the star formation simulations had a total of 87040 particles each, with 24576 for the gas, 21504 for the stellar disk, 8192 for the bulge, and 32768 for the halo. The gas was initially distributed like the stellar disk, and accounted for 12.5 per cent of the total disk mass. In simulation units, the gas had a fixed sound speed $c_{\mathrm{s}}=0.0966$, mimicking a warm ISM with $T \sim 10^{4} \mathrm{~K}$.

These calculations were run with a SPH code featuring adaptive smoothing and individual particle timesteps. The smoothing radius $h_{i}$ of each gas particle was set so that a sphere of radius $2 h_{i}$ contains exactly $N_{\text {smooth }}=40$ gas particles. A modified Courant condition with $\mathcal{C}=0.25$ was used to determine individual particle timesteps, which in some cases were as small as $\Delta t_{i}=1 / 32768$ time units. The artificial viscosity parameters appearing in A3 were $\alpha=1.0$ and $\beta=2.0$. Gravitational forces were calculated using a modified tree code with Plummer smoothing (Aarseth 1963) and a smoothing length of $\epsilon=0.0125$. Collisionless particles were integrated with a fixed time-step of $\Delta t=1 / 512$ time units. Including the energy radiated (or absorbed) by the gas in order to maintain a constant temperature, energy was conserved to about 0.5 percent through the final merger of the galaxy models. Depending on the star formation parameters, each simulation took between 126 and $245 \mathrm{hr}$ on a $1 \mathrm{GHz}$ processor; the run without star formation was the slowest since its gas attained the highest densities.

In practice, the limited resolution of computer simulations introduces effects which complicate the interpretation of numerical experiments. Smoothing over a sphere of radius $2 h_{i}$ inevitably suppresses structures on smaller scales. The gas disks used in this paper provide good examples; for the simulation parameters given above, only half of the disk particles have smoothing radii $h_{i}$ less than the vertical scale height $z_{\text {disk }}$ ! Clearly, the vertical structure of these disks is not resolved - and the same is true for most SPH simulations of interacting galaxies published to date. In this circumstance, SPH underestimates $\rho_{\mathrm{g}}$ close to the midplane, and overestimates $\rho_{\mathrm{g}}$ far from the midplane.

\section{APPENDIX C: ISOLATED DISK MODELS}

To better characterize the behavior of the galaxy models used in the simulations of NGC 4676, I ran some test calculations of isolated disks. These can be used to establish the baseline rate of star formation in non-interacting disks, and to examine the radial distribution of star formation for various choices of $n$ and $m$.

Fig. C1 shows global star formation rates for isolated disk models like the ones used in the NGC 4676 simulations. As in Figs. 2 and 5 the solid lines show results for the density-dependent rule $\left(n=1.5, m=0, C_{*}=0.025\right)$ while the broken lines show results for shock-induced rules (dashed line: $n=1, m=0.5, C_{*}=0.5$; dotted line: $n=1$, $\left.m=1, C_{*}=0.25\right)$. Since the last of these yields a very low baseline rate, an additional calculation was run with $C_{*}$ increased by a factor of 8 (dot-dashed line: $n=1, m=1$, $\left.C_{*}=2.0\right)$. In every case, the isolated disk models proved stable for the duration of the calculation. Apart from the brief initial bursts in the shock-induced models due to imperfect initialization of gas velocities, the only trends seen are slight declines in global star formation due to gradual depletion of the gas.

Given the rather stable behavior of the isolated disk models, it's reasonable to measure radial star formation profiles by counting stars formed in concentric annuli. Fig. C2 plots $\dot{\Sigma}_{*}$ as a function of cylindrical radius. Much as expected, the density-dependent rule yields a good approximation to the standard Schmidt law with index 1.5. Less predictably, the shock-induced rules also approximate Schmidttype laws, albeit with somewhat shallower slopes. This outcome must depend on the dynamical structure of the 


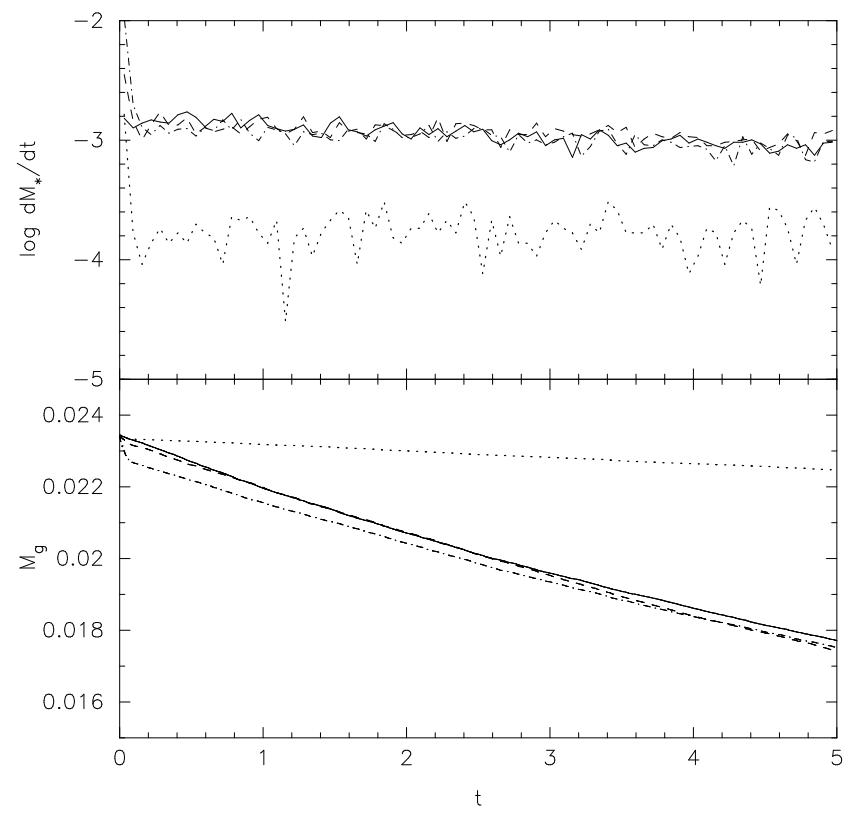

Figure C1. Global star formation in isolated disk simulations. Line types are identical to those in Fig. 2 in addition, the dotdashed line is $n=1, m=1, C_{*}=2.0$. Note that here the top panel shows $\log \dot{M}_{*}$. The simulations using shock-induced star formation produce brief initial bursts, but settle down promptly; no other features are seen in these plots.

disks; for a hypothetical and rather extreme example, a bar-unstable disk would presumably yield different star formation profiles before and after the bar formed. A shockinduced model in which the average shock strength is independent of $R$ should yield a Schmidt-type law with an index of unity, regardless of the chosen value of $m$. Here, however, the shock-induced models with $m=1.0$ appear to yield slightly steeper $\dot{\Sigma}_{*}$ profiles than does the model with $m=0.5$, hinting that the shocks are somewhat stronger near the center of this disk.

\section{REFERENCES}

Aarseth, S.J. 1963, MNRAS 126, 223

Alonso-Herrero, A., Rieke, G.H., Rieke, M.J., \& Scoville, N.Z. 2000, ApJ 532, 845

Barnes, J.E. 1992, ApJ 393, 484

Barnes, J.E. 1998, Galaxies: Interactions and Induced Star Formation, eds. D. Friedli, L. Martinet, D. Pfenniger (Springer-Verlag: Berlin), p. 275

Barnes, J.E. 2002, MNRAS 333, 481

Barnes, J.E. \& Hernquist, L. 1996, ApJ 471, 115

Burbidge, E.M. \& Burbidge, G.R. 1961, ApJ 133, 726

de Grijs, R., Lee, J.T., Clemencia Mora Herrera, M., Fritze-

v. Alvensleben, U., Anders, P. 2003, New Astr. 8, 155

Dehnen, W. 1993, MNRAS 265, 250

Freeman, K.C. 1970, ApJ 160, 811

Elmegreen, B.G. \& Efremov, Y.N. 1997, ApJ 480, 235

Gerin, M., Combes, F., \& Athanassoula, E. 1990, AAP, 230, 37

Gerritsen, J.P.E. \& Icke, V. 1997, A\&A 325, 972

Gilbert, S.J. \& Sellwood, J.A. 1993, Numerical Simulations

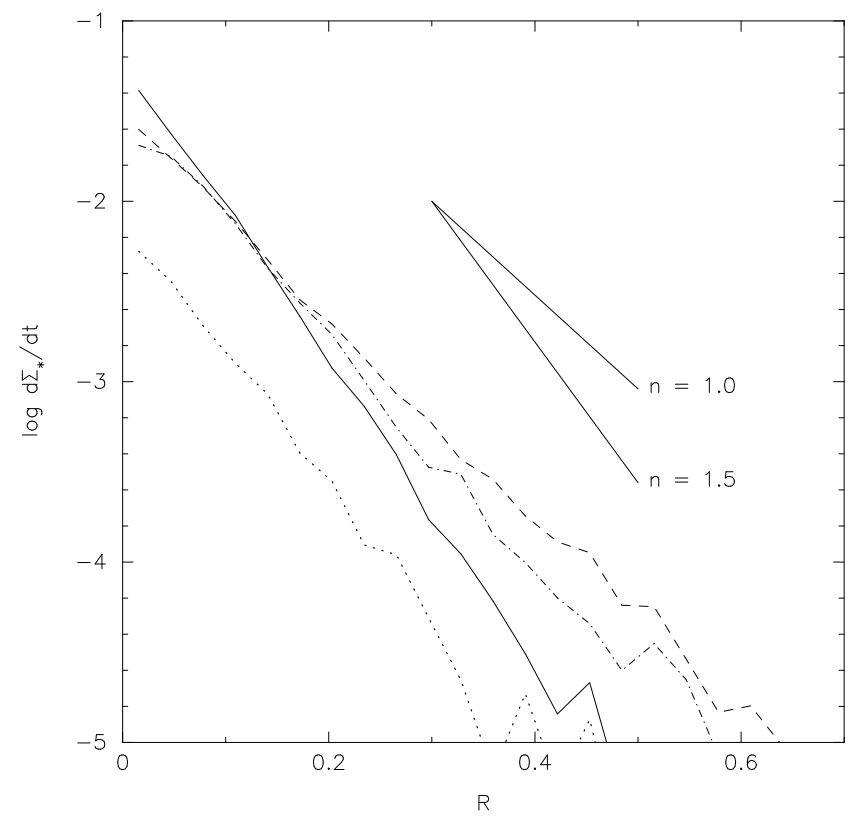

Figure C2. Radial star formation profiles for isolated disk simulations. Line types are identical to those in Fig. [C1 The two labeled lines correspond to Schmidt laws with the indicated values of $n$.

in Astrophysics, ed. J. Franco et al. (Cambridge University Press: Cambridge), p. 131

Gingold, R.A. \& Monaghan, J.J. 1977, MNRAS 181, 375

Hernquist, L. 1990, ApJ 356, 359

Hernquist, L. \& Katz, N. 1989, ApJS 70, 419

Hibbard, J.E. 1995, PhD thesis, Columbia University

Hibbard, J.E. \& van Gorkom, J.H. 1996, AJ 111, 655

Hibbard, J.E. \& Yun, M.S. 1996, AJ 118, 162

Jog, C.J. \& Solomon, P.M. 1992, ApJ 387, 152

Katz, N. 1992, ApJ 391, 502

Kennicutt, R.C. 1998, ApJ 498, 541

Kormendy, J. \& Sanders, D.B. 1992, ApJ 390, L53

Lucy, L. 1977, AJ 82, 1013

Mihos, J.C., Richstone, D.O., \& Bothun, G.D. 1991, ApJ 377,72

Mihos, J.C., Richstone, D.O., \& Bothun, G.D. 1992, ApJ 400, 153

Mihos, J.C., Bothun, G.D., \& Richstone, D.O. 1993, ApJ 418, 82

Mihos, J.C. \& Hernquist, L. 1994a, ApJ 437, 611

Mihos, J.C. \& Hernquist, L. 1994b, ApJ 431, L9

Mihos, J.C. \& Hernquist, L. 1994c, ApJ 437, L47

Mihos, J.C. \& Hernquist, L. 1996, ApJ 464, 641

Mirabel, I.F., Vigroux, L., Charmandaris, V., Sauvage, M., Gallais, P., Tran, D., Cearsky, C., Madden, S.C., \& Duc, P.-A. 1998, AsAp 333, L1

Monaghan, J.J. 1992, ARA\&A 30, 543

Monaghan, J.J. \& Lattanzio, J.C. 1985, AstrAp 149, 135

Negroponte, J. \& White, S.D.M. 1983, MNRAS 205, 1009

Noguchi, M. 1991, MNRAS 251, 360

Olson, K.M. \& Kwan, J. 1990, ApJ 361, 426

Sanders, D.B. \& Mirabel, I.F. 1996, ARA\&A 34, 749

Schmidt, M. 1959, ApJ 129, 243

Schweizer, F. 1990, in Dynamics and Interactions of Galax- 
ies, ed. R. Wielen (Springer: Berlin), p. 60

Scoville, N.Z., Sanders, D.B., \& Clemens, D.P. 1986

Shu, F.H. 1992, The Physics of Astrophysics, Vol. II (University Science Books: Mill Valley)

Sotnikova, N.Ya. \& Reshetnikov, V.P. 1998, AstL 24, 73

Spitzer, L. 1942, ApJ 95, 329

Springel, V. 2000, MNRAS 312, 859

Stockton, A. 1974, ApJ 187, 219

Theys, J.C., Spiegel, E.A., \& Toomre, J. 1972, PASP 84, 851

Toomre, A. 1977, The Evolution of Galaxies and Stellar Populations, eds. B. Tinsley \& R. Larson (Yale University Obs.: New Haven), p. 401

Toomre, A. \& Toomre, J. 1972, ApJ 178, 623

Tremaine, S., Richstone, D.O., Byun, Y.-I., Dressler, A., Faber, S.M., Grillmair, C., Kormendy, J., Lauer, T.R. 1994, AJ 107, 634

van den Bergh, S., Abraham, R.G., Ellis, R.S., Tanvir, N.R., Santiago, B.X., Glazebrook, K.G 1996, AJ 112, 359

Vigroux, L., Mirabel, F., Altieri, B., Boulanger, F., Cesarsky, C., Cesarsky, D., Claret, A., Fransson, C., Gallais, P., Levine, D., Madden, S., Okumura, K., \& Tran, D. 1996, AsAp 315, L93

Vorontsov-Vel'yaminov, B.A. 1958, Soviet Astr-AJ 2, 805

White, S.D.M. 1979, MNRAS 189, 831

Whitmore, B.C. \& Schweizer, F. 1995, AJ 109, 960

Yun, M.S. \& Hibbard, J.E. 2001, ApJ 550, 104

Xu, C., Gao, Y., Mazzarella, J., Lu, N., Sulentic, J.W., \& Domingue, D.L. 2000, ApJ 541, 644

Zepf, S.E. \& Ashman, K.M. 1993, MNRAS, 264, 611 